\title{
NEOGEOGRAFÍA: ALGO MÁS QUE CARTOGRAFÍA ACCESIBLE
}

José CORTIZO ÁLVAREZ (Coordinador) (jose.cortizo@,unileon.es)

Departamento de Geografía y Geología. Universidad de Léon

Como muy bien canta nuestro poeta Pablo Guerrero, el mapa no es el camino. Algo similar, aquello de que el mapa no es el territorio, parece ser que gritó el lingüista Alfred Korzybski, en la Primera Guerra Mundial, tras caer en una trinchera que no figuraba en sus mapas.

Así pues, ¿todo está en los mapas? Indudablemente, no. Sin embargo, el mapa ha jugado un papel vital en el desarrollo de la humanidad, como creemos que está sobradamente demostrado. Sin entrar siquiera en una somera revisión de lo que ha supuesto el mapa y en la historia de la Cartografía, nuestra impresión es que, después de un periodo de aparente indiferencia ante lo que podemos considerar como memoria gráfica del espacio, el mapa ha recobrado una importancia creciente, que trasciende el mundo académico, alcanza al público general y se asoma a los medios de comunicación.

En efecto, temas como el Big Data, Smart cities, la cartografía inteligente, la cartografía de los delitos, noticias sobre los mapas catastrales o referencias a cómo el geomárketing y los mapas ayudan en la elección de locales comerciales, son cada vez más frecuentes en nuestra prensa escrita. Es más, ya en 2009 un semanario decía de manera explícita que un concepto casi tan antiguo como la humanidad, el mapa, será el que genere grandes negocios en el futuro (EL PAÍS SEMANAL, 7-junio-2009).

Gran parte de la responsabilidad de este auge está en los avances tecnológicos, en las llamadas Tecnologías de la Información y Comunicación (TIC), en paralelo con el masivo tratamiento digital de la información geográfica (IG). En este contexto, la respuesta de los organismos productores de cartografía ha 
sido poner a disposición del público esa información geográfica. Por su parte, con el aporte de las TIC y de las redes sociales (web 2.0) se ha hecho posible la interacción con esa información. En gran medida, podemos decir que de la conjunción de estos elementos (TIC e IG) han surgido las Nuevas Tecnologías de la Información Geográfica (NTIG).

El mapa, así pues, ha recobrado protagonismo y en torno al mismo pensamos que deberían discurrir hoy buena parte de las discusiones metodológicas y conceptuales en el seno de nuestra disciplina, de la Geografía; sin embargo, el «problema» de la Geografía ha sido su lenta respuesta a la nueva realidad, la cual parece imponer el desarrollo de un nuevo paradigma que dé cobertura a nuevos aspectos relacionados con lo más esencial de la disciplina, con la información geográfica, su análisis y su representación. En definitiva, a lo que ha venido a denominarse Neogeografía.

Esa falta de referente queda comentada en varios artículos de este monográfico. Ahora bien, ¿qué debe tener en cuenta ese nuevo paradigma? En nuestro criterio, debe considerar y aunar elementos conceptuales con herramientas, abordando temas como son la difusión y acceso masivos a la información espacial (visores web), la interacción con la misma (cartografía voluntaria), la explotación de los datos inherentes a esa información por diversos agentes y múltiples finalidades (Big Data, app) o la privacidad (geolocalización).

Desde nuestro punto de vista, el marco teórico-metodológico en formación debería dar respuesta a los nexos que configuran el circuito integrado por: Información - Geografía - Cartografía - Neogeografía.

No obstante lo dicho, no todo es barbecho. En esta línea, tenemos, entre otros, un buen precedente en el texto de Warf y Sui (2010), estimulante ya desde su propio título (From GIS to neogeography: ontological implications and theories of truth) y en el que se hacen eco de esa certeza de que la Neogeografía plantea retos epistemológicos a la teoría dominante. En el texto realizan toda una declaración de principios en cuanto a lo que subyace en el éxito de la Neogeografía: La popularidad de la Neogeografía refleja la naturaleza cada vez más «fractalizada» de la vida social y espacial en el capitalismo contemporáneo, un mundo en el cual la estructura y el significado de la geografia han sido profundamente transformados bajo los efectos simultáneos de la globalización y de las crecientes tecnologías de la información (2010: 205).

En un plano general, el sociólogo Manuel Castells lleva tiempo poniendo en evidencia en diversas publicaciones el papel que ejercen en nuestra sociedad lo que él denomina «Galaxia internet», la sociedad de la información o la configuración de la sociedad red, entre otros elementos relevantes relacionados 
con el tema que planteamos. Así, por ejemplo, en su discurso de investidura como Doctor Honoris Causa por la Universidad de León, Castells hizo una llamada de atención, de manera específica, a la problemática de la transformación de nuestras sociedades, a partir de los procesos de cambio tecnológico y social que caracterizan la era de la información (CASTELLS, 2004: 9).

Por su parte, desde la Geografía, Horacio Capel incorpora en su obra Filosofia y ciencia en la geografía contemporánea. Una introducción a la Geografia un capítulo sobre Nuevas geografias y neogeografía. En el mismo, Capel hace un repaso muy interesante de los elementos fundamentales en esta reciente corriente de la disciplina, como son la vinculación entre el conocimiento geográfico y los avances técnicos o los nuevos agentes en la geografía y la cartografía; plantea, igualmente, la pertinencia de un nuevo paradigma o, cuanto menos, que los geógrafos participen activamente en la discusión de los problemas actuales y en la construcción del futuro (CAPEL, 2012.

Por lo demás, algunos de los colaboradores de este número también han hecho interesantes aportaciones en el marco teórico, como son los casos de Gustavo D.Buzai (2014a, 2014b) y Antonio Moreno (2013).

Por lo que se refiere al monográfico que presentamos, el número 27 de $\boldsymbol{P o}$ lígonos. Revista de Geografía, de 2015, está dedicado a cuestiones en pleno proceso de formación y desarrollo, que involucran a elementos sustanciales de la Geografía y que incumben tanto a la esencia de la disciplina como al método y a las herramientas. El punto de partida de este monográfico sobre Neogeografía fue no dar carta de naturaleza al nuevo marco en el que se desenvuelve la Geografía, en gran parte asociado a la tecnología, sin, al menos, cuestionar sus bases y su consistencia.

La Neogeografía no es, en estos momentos, un tema totalmente nuevo. Sobre el mismo se ha reflexionado y se han escrito interesantes trabajos y hay numerosa bibliografía fácilmente disponible en la red de Internet.

Partimos del hecho de que estamos ante un tema que plantea diversas facetas y que se presenta como multidimensional dentro de nuestra disciplina, aunque de manera simplificada y como pares no necesariamente excluyentes, podamos hacer referencia a los siguientes elementos subyacentes al mismo:

a. El análisis espacial como metodología frente a los instrumentos (mapa) como herramienta.

b. El avance desde el academicismo frente a las aportaciones «voluntarias» $\mathrm{y}$ «distribuidas». 
Todo lo anterior, además, sostenido por los avances técnicos y sociales que se apoyan en la red de Internet y en las llamadas "redes sociales", la web 2.0. La creación y la generalización de visores de cartografía con aplicaciones interactivas son uno de los elementos visibles más característicos de este movimiento.

En principio, asumimos que la Neogeografia hace referencia, pero no sólo, a las técnicas y herramientas geográficas utilizadas para actividades personales o utilizadas por personas o grupos de no expertos, de manera informal (TURNER, 2007).

No es nuestra intención discutir aquí en torno a la definición de la Neogeografía, sino que en este monográfico planteamos una serie de textos que abordan aspectos puntuales que, a nuestro juicio, forman parte del contexto en que se desenvuelve la evolución de la propia disciplina geográfica. A partir de esta premisa, en este número de la revista buscamos una reflexión acerca del alcance del uso de la información geográfica vinculada a las Tecnologías de la Información.

En este sentido, más que una colección de trabajos en torno al término y al concepto de Neogeografía, hemos tratado de reunir una serie de textos que cubran un espectro más amplio, que va desde la reflexión teórica hasta el análisis de alguna de las herramientas e información que están llamadas a jugar un papel de primer orden en nuestra disciplina, sobre todo por su disponibilidad abierta. Todo ello con la intención última de abordar los pares no excluyentes antes citados.

En este punto, consideramos que está fuera de toda duda la gran cantidad generada y la fuerte expansión de la información geográfica en la sociedad actual, hasta el punto de que podemos hablar del amplio consumo de esa información por diversos medios; de manera particular y generalizada a través de la red de Internet.

En lo que se refiere a la información geográfica y su cartografía, recordemos que algunas de sus características básicas en la actualidad son: dinamismo en su actualización, uso informal, uso masivo, libertad de acceso, interacción, sensores móviles y, por encima de todas, geolocalización.

Desde el punto de vista tecnológico, en todo lo relativo a la información territorial han jugado un papel fundamental los visores cartográficos que instituciones y empresas han incorporado a sus páginas web. Estas aplicaciones han puesto a disposición del público en general una cantidad ingente de información en bruto y también de cartografía elaborada. 
Metodológicamente y de manera práctica, las Infraestructuras de Datos Espaciales (IDEs) han sido uno de los grandes motores de este movimiento de difusión/acceso a la información espacial. Cuenta con los precedentes de los movimientos «Open», sobre todo del OPEN GEOSPATIAL CONSORTIUM (creado en 1994), cuya finalidad era la definición de estándares abiertos e interoperativos en los Sistemas de Información Geográfica y en la red de Internet. La Directiva europea INSPIRE (2007) ha dado carta de naturaleza a la interoperabilidad, la armonización de los conjuntos y servicios de datos espaciales, la normalización de la información geográfica, de su semántica y, fundamentalmente, a la difusión abierta de la información cartográfica.

En el contexto de las Tecnologías de la Información, el marco en el que se ha desarrollado la Neogeografía está definido por la conjunción de diversos factores ${ }^{1}$ :

1. Masividad en el acceso a Internet. La Web 2.0 es el elemento clave

2. Popularización del uso de dispositivos portátiles de comunicación (particularmente de los GPS)

3. Las nuevas comunidades digitales que se forman bajo algún tópico de interés particular

4. Abundante oferta de servicios públicos de productos de información, relacionada con las IDEs

5. Servicios geográficos básicos brindados por grandes empresas (tales como Google, Yahoo, Microsoft, Waze, Open Street Map o Foursquare, por citar algunos).

Muchos de los autores que abordan el tema de la Neogeografía señalan que una de sus características, puede expresarse en términos de «democratización de la información geográfica» o «socialización de la Geografía». En efecto, el acceso a la información geográfica y/o cartográfica mediante los visores pone la misma al alcance de un número potencial muy alto de personas. Por su parte, la posibilidad de interactuar y construir mapas a través de esos visores hace que la cartografía, en sentido laxo, deje de ser un campo exclusivo del personal formado profesionalmente en esta disciplina; pasa a ser un terreno en el que casi cualquier usuario puede construir un mapa ${ }^{2}$. En esta línea, es interesante, por ejemplo, la referencia que hace Joaquín Bosque en su texto de este monográfico a la wikificación del SIG.

\footnotetext{
${ }^{1}$ Adaptado de https://sites.google.com/site/geolocalitzacio/neogeografia )

2 Ibidem
} 
Si bien podemos hacer una valoración positiva de la situación que comentamos, con el acceso abierto a la información geográfica y cartográfica y la interacción con la misma (la Cartografía Voluntaria, CV), son múltiples las implicaciones que para nuestra disciplina plantea el nuevo escenario.

Entre las preguntas que surgen ante los cambios hay algunas como ¿cuáles son los efectos en la sociedad de esta disponibilidad creciente de información? ¿Qué repercusiones puede tener en la Geografía el hecho de que esta información sea además, en muchos casos, interactiva? ¿Cuáles son las consecuencias para la Cartografía? ¿Hasta qué punto es fiable la construcción voluntaria de la información geográfica y de su cartografía de manera interactiva a través de los visores web?

Los efectos, en el caso de la Cartografía, son claramente visibles y no siempre positivos. Son numerosos los ejemplos del uso, indiscriminado y sin fundamento semiológico, de formas, volumen y color cuya única explicación radica en el predominio del diseño frente a la ortodoxia de la semántica visual. De todos modos, el problema no es nuevo; ya en 1998 Tomás Cortizo ponía de relieve el mal uso del lenguaje visual aplicado a los gráficos y a la cartografía.

Por otro lado, además del mal uso del lenguaje visual, por desconocimiento, debemos tener presente el posible, y probable, problema de la calidad de la información geográfica construida en la web 2.0. Más allá de la simple representación, interpretando a WARF Y SUI (2010), ¿dónde queda la «verdad» cuando entre el no experto (el «geógrafo/cartógrafo voluntario») que genera sus datos y su «historia» (sus «geografías») existe una discrepancia con los criterios rígidos de los expertos en SIG?.

Pero no solamente hablamos de lenguaje y de calidad de la información; también hay otros elementos presentes y aparentemente aceptados sin discusión. Así, ¿por qué la proyección Mercator sigue siendo la utilizada por la mayor parte de los visores cuando representan el mapamundi?

Pero no todo es negativo o tiene connotaciones negativas. La creatividad puesta en movimiento por los «cartógrafos voluntarios» nos ofrece ejemplos, cuanto menos curiosos, de cómo los elementos más dispares han sido llevados al terreno cartográfico (o seudo-cartográfico) y que nos permiten viajar por territorios inventados, por el mundo de los cruasanes, las giras de los Rolling Stones, los desastres urbanísticos españoles o la localización (y contenidos) de los mensajes de Twiter.

En otros ámbitos, la actuación de estos voluntarios de la cartografía ha sido crucial en catástrofes naturales, como las derivadas de los terremotos sufridos 
por Haití o Nepal, en las que la «cartografía voluntaria» constituyó una ayuda fundamental en la distribución de recursos en zonas en las que la cartografía oficial era bastante deficiente (EL PAÍS, 12-03-2015); avala su aportación el hecho de que incluso el ejército nepalí recurrió a esta información espacial, la más actualizada. La cartografía voluntaria ha tenido otro exponente notable en la información sobre las infraestructuras y los focos de infección del ébola: Cuando llega una persona con ébola a un hospital, lo primero que le preguntan los médicos es dónde vive, quiénes son sus familiares, sus movimientos... Pero todos esos datos sin ponerlos en un mapa, serían inútiles (EL PAÍS, 30noviembre-2014).

Una cuestión relevante es que la evolución del uso de la información geográfica y de la cartografía voluntaria, así como muchas reflexiones sobre este tema, se han producido fuera y/o al margen del mundo estrictamente académico. En esta línea, la necesidad de que la Geografía como disciplina se abra a las nuevas perspectivas y busque su posición en el nuevo marco, que procure su adecuación a la nueva realidad de la información geográfica, es una conclusión compartida en los textos que prologamos.

En este punto, la red de internet toma un protagonismo de primer orden, principalmente en forma de blogs que recogen tomas de posición y opiniones, la mayor parte de las veces razonadas y razonables, pero también, en ocasiones, con un discurso incoherente. Lejos de hacer valoraciones sobre este medio y sus contenidos y, mucho menos, comparaciones con las aportaciones académicas, debemos reconocer que estas formas de expresión superan a las fuentes tradicionales en las que se ha basado la evolución de nuestra disciplina en que están muy pegadas a la realidad y tienen una gran su rapidez de respuesta; la inmediatez es parte de su esencia.

Para acabar con esta introducción, aunque no ha sido abordado de manera extensa en este monográfico (queda mencionado en los textos de Antonio Moreno y de Gerson Beltrán), hay un aspecto sobre el que quiero llamar la atención; es el tema de la geolocalización y la pérdida de privacidad que puede implicar.

A veces es considerado como un tema tangencial, pero en mi criterio no es menor, por cuanto el acceso a la información geográfica y la interacción con la misma mediante dispositivos móviles y fijos dependen con frecuencia de que el usuario revele o permita que se conozca su localización. En definitiva, en estos términos, en muchas ocasiones la disponibilidad de información espacial implica responder a la disyuntiva privacidad - servicios relacionados 
con la geoinformación, con lo que esto significa (ver y ser visto). Los mapas de localización de Tweets pueden servir de ejemplo a lo que comentamos ${ }^{3}$.

Entrando en el contenido de este monográfico, si bien resulta complejo hacer una clasificación de los textos que lo forman, hemos intentado estructurar el número ordenando los artículos según su contenido yendo, en la medida de lo posible, de lo general a lo particular.

Con sus matices, en los textos que prologamos hay confluencias en su contenido, lógicas, por otra parte. Así, se hace referencia en los mismos a: una nueva realidad con nuevas relaciones y que genera nueva información geográfica; las llamadas TIC tienen especial importancia en este escenario; la interacción es una característica añadida recientemente a la información espacial; el big data y la privacidad forman parte del telón de fondo del actual escenario; la necesidad de formalizar un nuevo paradigma en torno a la información territorial.

Abrimos el monográfico con una serie de trabajos que nos llevan a una reflexión general sobre el tema que nos ocupa. El nuevo contexto geotecnológico, la relación de la disciplina geográfica con la sociedad, las nuevas formas de producción y consumo de información espacial o la geosemántica espacial o el big data son algunos de los puntos abordados.

Otros textos del monográfico tienen una componente más aplicada y, en algún caso, incluso instrumental. Abordan temas más concretos, menos teóricos, pero no por ello menos importantes: la cartografia voluntaria y otras formas de participación ciudadana, la calidad de la cartografía, las prestaciones de los visores de cartografía a la hora de presentar el relieve, el papel de la información geográfica y de su gestión eficaz en las smart cities o las utilidades de nuevas fuentes de información sobre el territorio, como son el LIDAR.

En primer lugar, incorporamos un texto de Antonio Moreno Jiménez sobre la Sociedad de la Geoinformación y conducta espacial del ciudadano como nuevos desafíos para la Geografía.

Dando por hecho que las transformaciones que está conociendo la información geográfica y su tratamiento tienen un carácter revolucionario, el autor se centra en la conducta espacial del individuo proponiendo un nuevo concepto, el Entorno Personal Próximo (EPP), en el que el protagonismo corresponde a la interacción y las relaciones del individuo. El contexto inmediato de este

\footnotetext{
3 TweetMap AlPHA. A Sample Big Geodata Exploration Tool. Powered by MapD and WorldMap. Véase http://worldmap.harvard.edu/tweetmap/
} 
EPP es la sociedad de la geoinformación (SGI); es el marco que define conceptualmente el espacio digital, el ciberespacio, en el que el individuo, conscientemente o no, se mueve.

Nos plantea Antonio Moreno, entre otras cuestiones, la necesidad de «pensar nuevos espacios», como es el EPP, y hacerlo mediante la puesta en escena de nuevas herramientas ontológicas. En este nuevo escenario, el EPP se podría tomar como una herramienta metodológica para analizar el territorio.

Por su parte, Gustavo D. Buzai, en su trabajo Geografía global y Neogeografía. La dimensión espacial en la ciencia y en la sociedad, mediante un enfoque a distintas escalas, nos sitúa ante un nuevo ciclo en la evolución de la disciplina geográfica en cuya historia, según este autor, estamos ante el comienzo de una nueva etapa.

En el nuevo escenario, adquiere una importancia singular el uso de las Tecnologías de la Información Geográfica (TIG). De hecho, este marco geotecnológico es el contexto en el que se insertan, si vamos de lo general a lo particular, las relaciones entre ciencia y sociedad, el pensamiento geográfico actual, el análisis geográfico y las nuevas formas de la creación de cartografía, definidas por el voluntarismo y su carácter amateur.

A nuestro juicio, son tres los elementos clave en torno a los cuales gira el texto de Buzai: la «tercera globalización», basada en los flujos de datos digitales; la Geografía Global, como orientación científica; la Neogeografía, como procedimiento social.

Diego Cerda Seguel, en su artículo Mapas digitales y sociedad: Geosemántica social, el poder del sentido de lugar, nos sitúa ante una reflexión centrada en la confluencia entre lo geográfico y lo social basándose, de manera concreta, y entre otros argumentos, en el desarrollo tecnológico que permite la interacción con la información geográfica.

Para este autor, el marco teórico definido por la geosemántica social hace referencia al análisis de las nuevas formas de "apropiación" de los espacios geográficos, en las que la geotecnología tiene un papel primordial: permite realizar anotaciones, discusiones, valoraciones y, no menos importante, compartir la información de carácter territorial.

El acceso abierto a la información espacial y la posibilidad de actuar sobre la misma mediante operaciones como la que describe el autor, la georreferenciación, da a cualquier usuario la capacidad de intervenir a través de las ano- 
taciones. En su trabajo, Diego Cerda hace un repaso de algunas de las aplicaciones que hacen posible esta interacción: Waze, Four Square, Open Street Map y Google.

En la línea de lo anterior, uniendo lo social y lo tecnológico, la disponibilidad abierta de la información geográfica, es decir, la instalación del mapa digital en los social-media explica el surgimiento de la Neogeografía.

La geolocalización social es el tema que estudia Gerson Beltrán en su artículo. Parte de la hipótesis de la evolución de la sociedad hacia un «estado líquido», para cuyo análisis la Geografía debe evolucionar desde el anclaje que mantiene en el punto de vista «industrial» en vigor hacia otra perspectiva en la que se otorgue el peso que le corresponde en la actualidad a la tecnología y a lo digital, hacia la Neogeografía o Geografía 2.0. Esto le da pie para su argumentación, introduciendo la variable del desarrollo de la Neogeografía y sus aportaciones.

La geolocalización es uno de los conceptos básicos en la Neogeografía y Gerson Beltrán lo vincula a las redes sociales señalando que son las dos caras de la misma moneda, conformando la base de la geolocalización social. Las Tecnologías de la Información Geográfica son el soporte instrumental de la geolocalización social, en la que cobran protagonismo los dispositivos tecnológicos móviles como germen de nuevas formas de relación socio-espacial.

Este concepto de geolocalización social abre las puertas a nuevas aplicaciones y a nuevas reflexiones, no cerradas, en pleno desarrollo actualmente y apuntadas por el autor y que incluyen el geomárketing, el geoposicionamiento emocional (neuromárketing), el big data o el problema de la privacidad.

En su texto La vía ecléctica de producción y consumo de datos espaciales, Jorge del Rio parte de la premisa de la irrupción masiva de los datos espaciales, que ha generado importantes cambios y a un ritmo desconocido anteriormente. Los paradigmas geográficos vigentes no han dado respuesta a la nueva situación, abocando a lo que el autor denomina «el atasco actual»en el análisis de nuestra disciplina.

Esa entrada masiva está intrínsecamente asociada al uso de la tecnología, particularmente de las redes sociales, aplicada a los procesos de creación, gestión, difusión y uso de los datos geográficos. En este sentido, ante la nueva situación y la respuesta de nuestra disciplina, en el texto se aborda la vía ecléctica, es decir, la convivencia de distintos paradigmas mediante los cuales podemos analizar los cambios generados. 
Después de revisar la situación actual, el autor nos propone el proceso analítico jerárquico AHP como modelo de análisis. En este marco se revisan los paradigmas que, dentro del contexto de los datos (socioeconómico, industrial y técnico, individual), mejor explican los nuevos fenómenos en la Geografía actual. En su línea de razonamiento, los procedimientos de producción y consumo de datos ocupan un lugar importante y a los mismos dedica una parte de su artículo, tomando las que denomina "geocomunidades" como hilo conductor.

No deja de resultar curioso encontrarse con una noticia de prensa en la que se dice que «la cartografía ha llegado en auxilio del big data». Según esta noticia, los Sistemas de Información Geográfica permiten la recopilación y organización de los datos, de todo tipo, para convertirlos en información útil para «todas las ramas de la investigación científica, económica y social» (EL PAÍS, 30-11-14).

A este tema dedica un texto Joaquín Bosque Sendra, con el título de Neogeografía, BIG data y TIG: problemas y nuevas posibilidades.

Partiendo del hecho constatado de la abundancia creciente de los datos espaciales, así como su fácil acceso y disponibilidad, superado un gran problema como era el de la disponibilidad de información, en su trabajo Bosque nos pone ante cuestiones muy importantes, ya que además de su abundancia, ha cambiado el perfil y la cualidad de los datos, manteniendo su componente espacial: movimientos de personas, acceso a lugares, entre otros.

En este sentido, desde nuestra perspectiva, el texto abre unas interesantes vías de reflexión con dos puntos de partida: a) señalando que los nuevos datos muestran procesos y no estructuras «estables» y b) al plantear la necesidad de elaborar nuevos modelos de análisis que respondan precisamente a la dinámica de esos procesos.

La reflexión acerca del papel de los «nuevos creadores» y «nuevos protagonistas» del conocimiento geográfico es abordada por Jordi Guimet en su texto Crowsourcing participativo institucional. Información Geográfica Voluntaria en la Administración Pública. Ejemplos. Este tema de la Información Geográfica Voluntaria (VGI) o «Geo-crowdsourcing» se enmarca en el contexto del binomio información espacial-redes sociales (web 2.0); en el centro de la cuestión, el acceso interactivo a la información geográfica.

En este trabajo se analizan tres ejemplos de geografía voluntaria. El primero se basa en la Plataforma de Recursos de Información, en la que se basan algunos municipios catalanes para la visualización y la interacción de su información geográfica; el segundo trata el mantenimiento y mejora de la Base de 
Equipamientos Públicos; el tercero, finalmente, recoge la actualización del callejero por parte del Instituto Cartográfico y Geológico de Cataluña.

El problema de la calidad, inherente a este tipo de procedimientos, queda mencionado en este trabajo en un breve apartado: resolución, certeza y actualización son, entre otros, posibles problemas asociados a la cartografía voluntaria.

La vertiente de la calidad de los datos cartográficos y de su gestión, en el contexto del uso amplio de la tecnología de acceso y manipulación de la información espacial, ha estado en el fondo de las preocupaciones en la configuración de este monográfico. A nuestro modo de ver, las herramientas de la red y la «nube» permiten un acceso masivo a la información geográfica/cartográfica y el uso de la misma para la creación de mapas y aportan ventajas indudables en la línea de lo que subyace en la Neogeografía, la democratización de la información geográfica; no obstante, también es cierto que conllevan cierto riesgo, el de la pérdida de calidad del producto final, del mapa, tanto en lo semiológico como en lo geográfico.

En relación con el tema de la calidad de los datos geográficos, Francisco Javier Ariza aporta un trabajo centrado en los riesgos que existen para la misma: La construcción descentralizada de datos espaciales: riesgos para la calidad de la información geográfica.

Este texto nos lleva a un repaso muy interesante de los principales riesgos derivados de la construcción descentralizada de la información geográfica y que tiene que ver con: su producción, acceso, metadatos, interoperabilidad, metadatos, trazabilidad y metacalidad y, finalmente, la preservación.

Está claro que este trabajo nos lleva a otro nivel de mayor calado que el que se asocia a la mera interacción con la información geográfica a través de visores web, la "cartografía amateur". El autor nos llama la atención acerca de que estos riesgos ya existían, pero se han visto agudizados con el acceso y manipulación masiva de los datos cartográficos. Normalización de la información geográfica y formación de los participantes son algunas de las soluciones que se plantean.

Enlazando con la cuestión de la calidad de la información geográfica, un problema no menor, en medio de la proliferación de los visualizadores vinculados a las Infraestructuras de Datos Espaciales, es el de la visualización del relieve. Mejor dicho, del pseudorrelieve, como bien señalan Miguel Ángel Bernabé-Poveda, María Ester González y Ángeles Castejón en su texto El pseudorrelieve en los visualizadores de las IDE de España. 
Los autores hacen un repaso a los visores de las IDEs de las Comunidades Autónomas y acaban con la referencia a un test que han desarrollado en internet, cuyos resultados nos muestran que la pretensión de informar acerca de la configuración del relieve no siempre es conseguida de manera total y acertada.

Mediante un numeroso aparato gráfico, nos ponen en evidencia muchas de las carencias de estos visores, de modo particular cuando pretenden hacer visible las tres dimensiones intrínsecas a la topografía del terreno.

En nuestra opinión, problemas de diseño en la presentación de la información geográfica expuesta en las IDEs llevan a que aquello que debería la percepción del relieve acabe siendo la percepción de un falso relieve, en el que los cursos fluviales discurren sobre las líneas de cumbres, entre otras peculiaridades. No olvidemos que, en términos de representación cartográfica, trabajamos con lenguaje visual y percepción, y esta es siempre subjetiva.

Maite Zúñiga y otros cinco colegas firman el texto Generando opinión ciudadana en la cultura digital a través de los mapas en el que recogen una interesante experiencia, la derivada de la exposición «Zaragoza mapa a mapa: los servicios a tu lado» (octubre-diciembre de 2013). Esta intervención tiene su origen en proyectos anteriores basados en el proceso de poner la información geográfica al alcance de los ciudadanos de Zaragoza, particularmente aquella de escala más próxima a su entorno habitual, con la intención final de obtener la interacción entre ciudadano e información geográfica.

Entre otros aspectos relevantes, a lo largo de la exposición, la interacción de los visitantes mediante las redes sociales añade no solamente valoraciones cualitativas acerca de su contenido sino, lo que es más interesante, en mi criterio, nueva información acerca de la ciudad.

En este contexto de información geográfica relativa al entorno urbano, los geógrafos Eduardo Bustillo y Pablo Rodríguez nos entregan un trabajo también a la escala del ciudadano y muy relacionado con la información geográfica y las tecnologías de la comunicación: Los sistemas de información geográfica y las ciudades inteligentes.

La IG, los SIG y las TIC tienen un protagonismo especial en el mundo de las llamadas ciudades inteligentes (Smart cities), son su esencia. Esto queda muy bien manifestado en este trabajo, que pone el énfasis en algunas de las cuestiones básicas, como son, por un lado, el papel de la ciudad como generadora de datos y, por otro, el rol del ciudadano como consumidor y a la vez productor de información geográfica. 
Son numerosos los protagonistas implicados en todo ese proceso de generación, recogida, elaboración y representación de la información geográfica urbana: ciudadanos, administración (policía local, entre otros agentes), servicios ciudadanos (taxis, por ejemplo). Es ingente la información puesta en juego y numerosas, igualmente, las aplicaciones y utilidades que se pueden dar a la misma.

Aunque no queda explícito en el texto, desde un punto de vista práctico y aplicado pensamos que los geógrafos pueden tener en este campo un buen nicho de trabajo, dando sentido a la cita que incluimos al comienzo de esta presentación (un concepto casi tan antiguo como la humanidad, el mapa, será el que genere grandes negocios en el futuro).

Cerramos el monográfico con un texto sobre El PNOA-LIDAR-CyL-2010: condicionantes técnicos de su elaboración, limitaciones y potencialidades para su aprovechamiento, escrito por el geógrafo Óscar Odón Rodríguez.

El desarrollo tecnológico que hemos comentado y que está en la base de la difusión masiva de la información geográfica tiene otras vertientes, menos asequibles y accesibles, y también menos "visibles" (incluso invisibles), para el público general y también para buena parte de los profesionales no vinculados de manera directa con la información derivada de la teledetección.

En este contexto, el LIDAR (Light Detection and Ranging) está tomando protagonismo como una fuente importante para la información sobre el territorio. Por sus propiedades, nos permite capturar información acerca de la superficie de la tierra en las tres coordenadas $(\mathrm{x}, \mathrm{y}, \mathrm{z})$ con gran precisión, hasta el punto de que para ciertas aplicaciones podría sustituir al trabajo cartográfico tradicional (incluida la fotogrametría). Modelos de elevaciones (MDE) o clasificación de usos del suelo pueden ser algunas de sus aplicaciones.

En el texto se hace un repaso técnico del LIDAR asociado al Plan Nacional de Ortofotografía Aérea (PNOA), concretamente en lo relativo a Castilla y León, haciendo referencia a sus características principales, que tienen que ver con parámetros del sensor y del tratamiento de la información procedente de esa teledetección. El autor, además, nos muestra ejemplos de la calidad y exactitud de la información ofrecida del modelo del terreno y de la discriminación de usos del suelo en un entorno urbano.

El tratamiento de esta rica información no es, sin duda, fácil ni asequible al público (al menos no como estamos acostumbrados en los visores habituales); sin embargo, el trabajo de los responsables de la misma (CNIG, Junta de Castilla y León), al decir del autor, se encamina a que haya una mayor disponibilidad de la misma. 
A buen seguro, esta nueva fuente de datos espaciales nos proporcionará una buena base $\mathrm{o}$, al menos, un buen complemento para la información geográfica que, por el momento, manejamos de manera habitual.

Para finalizar, debemos señalar que la intención de este monográfico era plantear un momento de deliberación que se puede resumir en una pregunta: ¿estamos ante un cambio profundo en la Geografía, o se trata simplemente de otros métodos para otras preguntas, al decir de F.J. Tapiador?

Para acercarnos a una respuesta, y con la intención de mostrar que la Neogeografía es algo más que mapas (inteligentes), hemos reunido un conjunto de textos que pretenden aunar la perspectiva hecha desde el mundo académico con acercamientos al tema desde ámbitos no necesariamente universitarios. Dos mundos a veces percibidos como contrapuestos pero llamados a confluir.

Si hemos conseguido mover a la reflexión, es algo que compete valorar a los lectores interesados; por nuestra parte, nos damos por satisfechos con el trabajo realizado hasta la edición final de este monográfico: hemos aprendido mucho en el camino y esperamos haberlo transmitido.

A todos los autores que han colaborado paciente y desinteresadamente a que este número de Polígonos. Revista de Geografía saliera adelante, muchas gracias.

\section{BIBLIOGRAFÍA}

BUZAI, G.D. (2014a): «Neogeografía y sociedad de la información geográfica. Una nueva etapa en la historia de la Geografía». Boletín del Colegio de Geógrafos del Perú. 1, 1-12.

BuZAI, G.D. (2014b): «Desde la Geografía. Ciencia y tecnología en una sociedad de la información geográfica». GeoCritiq. 10 de junio de 2014.

CAPEL, H. (2012): Filosofía y ciencia en la geografía contemporánea. Una introducción a la Geografía. Barcelona, Ediciones del Serbal

CAstells, M. (2004): «La ciudad, entre lo local y lo global». Polígonos. Revista de Geografía, 14; 9-14

CORTIZO Álvarez, T. (1998): «La miseria de la construcción gráfica en la geografía española actual». Ería. Revista Cuatrimestral de Geografía, núm. 46, 148-163

Diario EL PAÍS: Semanal (7-junio-2009): http://elpais.com/diario/2009/06/07/eps/1244356018 850215.html

Mapas para cambiar el mundo. 30-11-14 http://elpais.com/elpais/2014/11/26/ciencia/1417005124 889841.html

Un mapa de nepal para llevar ayuda tras el terremoto. 12-03-2015 http://internacional.elpais.com/internacional/2015/05/07/actualidad/1431013845 664082.html 
GoODCHILD, M. F. (2007): «Citizens as sensors: the world of voluntereed geography». Geojournal 69 (4): 211-221.

MORENO JIMÉNEZ, A. (2013): «Entendimiento y naturaleza de la cientificidad geotecnológica: una aproximación desde el pragmatismo epistemológico», Investigaciones Geográficas, 60, 5-36.

http://rua.ua.es/dspace/bitstream/10045/34742/1/Investigaciones_Geograficas_6 0_01.pdf

OPEN GEOSPATIAL CONSORTIUM: http://www.opengeospatial.org/ogc/history

PARLAMENTO EUROPEO. Directiva Infrastructure for Spatial Information in Europe (INSPIRE) de 14 de marzo de 2007.

http://eurlex.europa.eu/LexUriServ/LexUriServ.do?uri=OJ:L:2007:108:0001:001 4:ES:PDF Transpuesta al marco legal español se lleva a cabo por medio de la Ley 14/2010, de 5 de julio, sobre las infraestructuras y los servicios de información geográfica en España (LISIGE) http://www.idee.es/espanol-lisige

TAPIADOR, F.J. (2006): «Las Tecnologías de Información Geográfica: Criticando al crítico. Una respuesta a Horacio Capel». Biblio $3 W$, Revista Bibliográfica de Geografia y Ciencias Sociales, Universidad de Barcelona, Vol. X, nº 646, 20 de abril de 2006.

http://www.ub.es/geocrit/b3w-646.htm

TURNER, A.J. (2006): Introduction to Neogeography. O`Reilly Media Inc.

TURNER, A.J. (2007) «Neogeography — Towards a Definition». Andrew Turner. Blog.

http://highearthorbit.com/neogeography-towards-a-definition

TweetMap ALPHA. A Sample Big Geodata Exploration Tool. Powered by MapD and WorldMap.

Véase http://worldmap.harvard.edu/tweetmap/

WARF, B. y SUI, D. (2010): «From GIS to neogeography: ontological implications and theories of truth». Annals of GIS, vol. 16, № 4, December, 197-209

Web Geolocalitació: https://sites.google.com/site/geolocalitzacio/neogeografia 